\title{
Conserved charges and black holes in the Einstein-Maxwell theory on $\mathrm{AdS}_{3}$ reconsidered
}

\author{
Alfredo Pérez, ${ }^{a}$ Miguel Riquelme, ${ }^{a, b}$ David Tempo ${ }^{a, c}$ and Ricardo Troncoso ${ }^{a}$ \\ ${ }^{a}$ Centro de Estudios Científicos (CECs), \\ Av. Arturo Prat 514, Valdivia, Chile \\ ${ }^{b}$ Departamento de Fúsica, Universidad de Concepción, \\ Casilla 160-C, Concepción, Chile \\ ${ }^{c}$ Université Libre de Bruxelles and International Solvay Institutes, \\ ULB Campus Plaine C.P.231, B-1050 Bruxelles, Belgium \\ E-mail: aperez@cecs.cl, riquelme@cecs.cl, tempo@cecs.cl, \\ troncoso@cecs.cl
}

ABSTRACT: Stationary circularly symmetric solutions of General Relativity with negative cosmological constant coupled to the Maxwell field are analyzed in three spacetime dimensions. Taking into account that the fall-off of the fields is slower than the standard one for a localized distribution of matter, it is shown that, by virtue of a suitable choice of the electromagnetic Lagrange multiplier, the action attains a bona fide extremum provided the asymptotic form of the electromagnetic field fulfills a nontrivial integrability condition. As a consequence, the mass and the angular momentum become automatically finite, without the need of any regularization procedure, and they generically acquire contributions from the electromagnetic field. Therefore, unlike the higher-dimensional case, it is found that the precise value of the mass and the angular momentum explicitly depends on the choice of boundary conditions. It can also be seen that requiring compatibility of the boundary conditions with the Lorentz and scaling symmetries of the class of stationary solutions, singles out a very special set of "holographic boundary conditions" that is described by a single parameter. Remarkably, in stark contrast with the somewhat pathological behaviour found in the standard case, for the holographic boundary conditions (i) the energy spectrum of an electrically charged (rotating) black hole is nonnegative, and (ii) for a fixed value of the mass, the electric charge is bounded from above.

KEYwords: Black Holes, Classical Theories of Gravity, Gauge-gravity correspondence

ARXIV EPRINT: 1509.01750 


\section{Contents}

1 Introduction 1

2 Reduced action principle: stationary solutions, integrability conditions and conserved charges

2.1 Compatibility of the boundary conditions with Lorentz and scaling symmetries 5

3 Black hole spectrum and Lorentz invariant boundary conditions: simplest and holographic choices

3.1 Static electrically charged black hole

3.2 Electrically charged rotating black hole

\section{Introduction}

In three-dimensional spacetimes, the fall-off of the electromagnetic field for a localized distribution of charge is very slow, and it then generates a strong backreaction in the asymptotic behaviour of the metric. Consequently, finding a suitable regularization for the energy in this class of instances turns out to be a hard nut to crack [1], even in the case of negative cosmological constant [2]. A similar situation occurs for General Relativity on $\mathrm{AdS}_{3}$ minimally coupled to scalar or two-form fields $[3,4]$ where, despite the asymptotic behaviour is relaxed as compared with the one of Brown and Henneaux [5], it is found that the conserved charges associated to the asymptotic symmetries become finite, and acquire terms that manifestly depend on the matter fields. Besides, the electrically charged black hole solution in the Einstein-Maxwell theory with negative cosmological constant, has been shown to exhibit somewhat pathological properties. Indeed, the energy is unbounded from below, and for a fixed value of the mass, the electric charge possesses no upper bound [2]. These unusual properties seem to suggest that the solution might be unstable, and it would also preclude its embedding within a suitable supergravity theory [6]. Note that if one takes into account that both, the black hole geometry and the Einstein-Maxwell Lagrangian are well behaved, this certainly becomes a very puzzling situation. In order to clarify the point, it is worth to stress that according to the action principle, the theory cannot be suitably understood without the knowledge of a precise set of boundary conditions. This issue is one of the main points of our work. The plan is as follows. In the next section we deal with stationary circularly symmetric solutions of General Relativity coupled to the Maxwell field on $\mathrm{AdS}_{3}$, and it is shown that a suitable choice of the asymptotic form of the electromagnetic Lagrange multiplier $\left(A_{t}\right)$, makes the action to attain an extremum provided it fulfills a nontrivial integrability condition. Hence, as in the case of scalar and 
two-form fields, for a generic choice of boundary conditions, the mass and also the angular momentum become automatically finite, and acquire explicit contributions from the matter field. In section 2.1, it is shown that requiring compatibility of the boundary conditions with the scaling symmetry of the class of stationary solutions, singles out a precise set of "holographic boundary conditions" that is described by an arbitrary function of a single variable. It can also be seen that if one further requires the holographic boundary conditions to be compatible with Lorentz symmetry, selects a very special subset that is parametrized just by a single arbitrary fixed constant. In section 3 we compare the global charges that are obtained in the case of an electrically charged rotating black hole solution for different choices of boundary conditions. It is shown that the standard result in [2] corresponds to the simplest choice of Lorentz invariant boundary conditions. Noteworthy, it is found that if the boundary conditions are compatible with both Lorentz and scaling symmetries, the energy spectrum of an electrically charged (rotating) black hole is nonnegative, and also, for a fixed value of the mass, the electric charge becomes bounded from above. We conclude with further remarks in section 4 .

\section{Reduced action principle: stationary solutions, integrability conditions and conserved charges}

Let us consider the electromagnetic field minimally coupled to General Relativity with negative cosmological constant in three spacetime dimensions. The action reads

$$
I=\int d^{3} x \sqrt{-g}\left[\frac{1}{2 \kappa}(R-2 \Lambda)-\frac{\epsilon_{0}}{4} F_{\alpha \beta} F^{\alpha \beta}\right],
$$

where the Newton constant $G$ and the AdS radius $l$ are defined through $\kappa=8 \pi G$ and $\Lambda=-l^{-2}$, respectively. The "vacuum permeability" $\epsilon_{0}$ has units of length, and is assumed to be fixed as $\epsilon_{0}=1$. A wide family of exact stationary circularly symmetric solutions has already been found in the literature [1, 2, 7-22]. Hereafter we deal with generic stationary circularly symmetric configurations, so that the spacetime metric can be written as

$$
d s^{2}=-\mathcal{N}(r)^{2} \mathcal{F}(r)^{2} d t^{2}+\frac{d r^{2}}{\mathcal{F}(r)^{2}}+\mathcal{R}(r)^{2}\left(\mathcal{N}^{\phi}(r) d t+d \phi\right)^{2},
$$

and the gauge field can be chosen to be given $b^{1}$

$$
A=\mathcal{A}_{t}(r) d t+\mathcal{A}_{\phi}(r) d \phi
$$

It is then clear that the form of (2.2) and (2.3) is mapped into itself under the action of a Lorentz boost in the " $t-\phi$ cylinder". It is also worth pointing out that the metric and the gauge field are invariant under scalings of the form

$$
r \rightarrow \lambda r, t \rightarrow \lambda^{-1} t, \phi \rightarrow \lambda^{-1} \phi,
$$

\footnotetext{
${ }^{1}$ Here the gauge field has been assumed to be stationary and circularly symmetric. Had we made the same assumption for the field strength, one would obtain a wider class of configurations, so that (2.3) corresponds to one of the two possible branches (see e.g., [22, 23]).
} 
provided

$$
\begin{aligned}
& \mathcal{F} \rightarrow \lambda \mathcal{F}, \quad \mathcal{R} \rightarrow \lambda \mathcal{R}, \quad \mathcal{A}_{\phi} \rightarrow \lambda \mathcal{A}_{\phi}, \\
& \mathcal{N} \rightarrow \mathcal{N}, \quad \mathcal{N}^{\phi} \rightarrow \mathcal{N}^{\phi}, \quad \mathcal{A}_{t} \rightarrow \lambda \mathcal{A}_{t} .
\end{aligned}
$$

These symmetries are then automatically incorporated in the reduced field equations that are obtained from the action (2.1) for stationary circularly symmetric configurations.

The reduced action principle is obtained by replacing the form of the metric and gauge field given by (2.2) and (2.3), respectively in (2.1), which reads

$$
I=-2 \pi\left(t_{2}-t_{1}\right) \int d r\left(\mathcal{N} \mathcal{H}+\mathcal{N}^{\phi} \mathcal{H}_{\phi}+\mathcal{A}_{t} \mathcal{G}\right)+B
$$

where $B$ stands for a suitable boundary term that has to be included in order to ensure that the action attains a bona fide extremum. It is then apparent that $\mathcal{N}, \mathcal{N}^{\phi}, \mathcal{A}_{t}$ are the Lagrange multipliers associated to the constraints, which read

$$
\begin{aligned}
\mathcal{H} & =-\frac{\mathcal{R}}{\kappa l^{2}}+4 \kappa \mathcal{R}\left(\pi^{r \phi}\right)^{2}+\frac{\left(p^{r}\right)^{2}}{2 \mathcal{R}}+\frac{\mathcal{F}^{2}\left(\mathcal{A}_{\phi}^{\prime}\right)^{2}}{2 \mathcal{R}}+\frac{\left(\mathcal{F}^{2}\right)^{\prime} \mathcal{R}^{\prime}}{2 \kappa}+\frac{\mathcal{F}^{2} \mathcal{R}^{\prime \prime}}{\kappa} \\
\mathcal{H}_{\phi} & =-p^{r} \mathcal{A}_{\phi}^{\prime}-2\left(\mathcal{R}^{2} \pi^{r \phi}\right)^{\prime} \\
\mathcal{G} & =-\partial_{r} p^{r}
\end{aligned}
$$

respectively, where the momenta $\pi^{r \phi}$ and $p^{r}$ are given by

$$
\pi^{r \phi}=-\frac{\mathcal{N}^{\phi \prime} \mathcal{R}}{4 \kappa \mathcal{N}} ; \quad p^{r}=\frac{\mathcal{R}}{\mathcal{N}}\left(\mathcal{A}_{\phi}^{\prime} \mathcal{N}^{\phi}-\mathcal{A}_{t}^{\prime}\right)
$$

Indeed, varying the action with respect to the Lagrange multipliers implies that

$$
\mathcal{H}=\mathcal{H}_{\phi}=\mathcal{G}=0,
$$

while the variation with respect to $\mathcal{A}_{\phi}, \mathcal{F}, \mathcal{R}$, yields the following field equations

$$
\begin{aligned}
\left(\mathcal{F}^{2} \mathcal{N} \mathcal{R} \mathcal{A}_{\phi}^{\prime}\right)^{\prime}= & 2 \mathcal{F}^{2} \mathcal{N} \mathcal{R}^{\prime} \mathcal{A}_{\phi}^{\prime}+\mathcal{R}^{2}\left(\mathcal{N}^{\phi} p^{r}\right)^{\prime} \\
\mathcal{R}^{\prime \prime}-[\log (\mathcal{N})]^{\prime} \mathcal{R}^{\prime}= & -\kappa\left(\mathcal{A}_{\phi}^{\prime}\right)^{2} \mathcal{R}^{-1} \\
\kappa\left[\mathcal{A}_{\phi}^{\prime 2} \mathcal{F}^{2}+\left(p^{r}\right)^{2}\right] \mathcal{R}^{-2}= & \left(\mathcal{F}^{2}\right)^{\prime \prime}+2\left[\mathcal{N}^{\prime \prime} \mathcal{F}^{2}+\frac{3}{2} \mathcal{N}^{\prime}\left(\mathcal{F}^{2}\right)^{\prime}+4 \kappa \mathcal{N}^{\phi \prime} \mathcal{R} \pi^{r \phi}\right] \mathcal{N}^{-1} \\
& +8\left(\kappa \pi^{r \phi}\right)^{2}+2 \Lambda
\end{aligned}
$$

The reduced action (2.6) then possesses an extremum $(\delta I=0)$ provided the variation of the boundary term $B$ is given by

$$
\delta B=-\left.\left(t_{2}-t_{1}\right) \delta Q(r)\right|_{r \rightarrow \infty},
$$

with

$$
\begin{aligned}
\delta Q(r)= & \frac{2 \pi}{\kappa}\left[\mathcal{N} \mathcal{F}\left(\mathcal{F}^{\prime} \delta \mathcal{R}-\delta\left(\mathcal{F} \mathcal{R}^{\prime}\right)-\kappa \frac{\mathcal{F}}{\mathcal{R}} \mathcal{A}_{\phi}^{\prime} \delta \mathcal{A}_{\phi}\right)+\mathcal{N}^{\prime}\left(\mathcal{F}^{2} \delta \mathcal{R}\right)\right] \\
& +2 \pi \mathcal{N}^{\phi}\left[p^{r} \delta \mathcal{A}_{\phi}+2 \delta\left(\pi^{r \phi} \mathcal{R}^{2}\right)\right]+2 \pi \mathcal{A}_{t} \delta p^{r}
\end{aligned}
$$


In order to integrate the variation of the boundary term one needs to know the behaviour of the fields in the asymptotic region. By virtue of the constraints and the field equations in (2.11) and (2.12), respectively, it can be seen that asymptotically $\mathrm{AdS}_{3}$ solutions possess the following fall-off:

$$
\begin{aligned}
\mathcal{R}^{2} & =r^{2}-\frac{\kappa l^{2}}{\pi}\left[h_{\mathcal{R}} \log \left(\frac{r}{l}\right)-\frac{f_{\mathcal{R}}}{2}\right]+\cdots \\
\mathcal{F}^{2} & =\frac{r^{2}}{l^{2}}-\frac{\kappa}{\pi}\left[\left(2 h_{\mathcal{R}}+\frac{1}{4 \pi}\left(q_{t}^{2}+q_{\phi}^{2}\right)\right) \log \left(\frac{r}{l}\right)+f_{\mathcal{F}}\right]+\cdots \\
\mathcal{N}^{\phi} & =N_{\infty}^{\phi}+\frac{\kappa}{2 \pi} N_{\infty}\left[\frac{l}{2 \pi} q_{t} q_{\phi} \log \left(\frac{r}{l}\right)-j\right] \frac{1}{r^{2}}+\cdots \\
\mathcal{N} & =N_{\infty}+\cdots \\
\mathcal{A}_{t} & =-\frac{1}{2 \pi}\left(q_{t} N_{\infty}+q_{\phi} l N_{\infty}^{\phi}\right) \log \left(\frac{r}{l}\right)+N_{\infty}^{\phi} \varphi_{\phi}+N_{\infty} \frac{\varphi_{t}}{l}-\Phi+\cdots \\
\mathcal{A}_{\phi} & =-\frac{q_{\phi} l}{2 \pi} \log \left(\frac{r}{l}\right)+\varphi_{\phi}+\cdots
\end{aligned}
$$

where the constants $h_{\mathcal{R}}, f_{\mathcal{R}}, f_{\mathcal{F}}, j, \varphi_{t}, \varphi_{\phi}, q_{t}, q_{\phi}$, are allowed to vary in the action principle, while $N_{\infty}, N_{\infty}^{\phi}$, and $\Phi$ stand for arbitrary constants without variation, whose value is kept fixed at the boundary. Here, "..." correspond to subleading terms that are irrelevant for the analysis.

It should be highlighted that the parameters that characterize the deformations of a spacelike surface at infinity, $N_{\infty}$ and $N_{\infty}^{\phi}$, have been manifestly incorporated in the asymptotic form of the electromagnetic potential $\mathcal{A}_{t}$. This has to be so in order to preserve the gauge fixing of the electromagnetic field under such deformations. In other words, this guarantees that the smeared canonical generator associated to the deformation parameters spans the Lie derivative of the gauge field along them. This procedure then improves the Hamiltonian by an additional contribution that comes from the $U(1)$ generator (see e.g. [24]). In $d \geq 4$ spacetime dimensions, the improvement just amounts to a proper gauge transformation that does not change the surface integrals associated to the canonical generators. However, in the three-dimensional case, due to the slow fall-off of the electromagnetic field, the improvement turns out to generate an improper gauge transformation that modifies the global charges in a nontrivial way. Hence, and remarkably, the logarithmic divergence in the boundary term that would arise from the original Hamiltonian precisely cancels out, without the need of any kind of regularization procedure. Therefore, for our prescribed fall-off in (2.14), the variation of the global charges (2.13) reduces to

$$
\delta Q=N_{\infty} \delta M-N_{\infty}^{\phi} \delta J-\Phi \delta q_{t}
$$

being manifestly finite. According to [25], eq. (2.15) allows to identify $\delta q_{t}, \delta J$ and $\delta M$ with the variation of the electric charge, the angular momentum, and the mass, respectively. The angular momentum directly integrates as

$$
J=j+\frac{l}{4 \pi} q_{t} q_{\phi}-q_{t} \varphi_{\phi}
$$


while the variation of the mass, given by

$$
\delta M=\delta\left[f_{\mathcal{R}}+f_{\mathcal{F}}+h_{\mathcal{R}}+\frac{1}{l}\left(q_{\phi} \varphi_{\phi}\right)\right]-\frac{1}{l}\left(\varphi_{\phi} \delta q_{\phi}-\varphi_{t} \delta q_{t}\right)
$$

implies a non trivial integrability condition involving $\varphi_{t}, \varphi_{\phi}, q_{t}, q_{\phi}$. Since the integrability condition is independent of the choice of $N_{\infty}$ and $N_{\infty}^{\phi}$, without loss of generality, it is convenient to express it in a manifestly Lorentz-covariant way, by assuming that the (conformal) boundary metric is given by the flat one, $\eta_{\mu \nu}=\operatorname{diag}\left(-l^{-2}, 1\right)$. Equation $(2.17)$ can then be written as

$$
\delta M=\delta\left[f_{\mathcal{R}}+f_{\mathcal{F}}+h_{\mathcal{R}}+\frac{1}{l}\left(q_{\phi} \varphi_{\phi}\right)\right]-\frac{1}{l} \varphi_{\mu} \delta q^{\mu}
$$

with $q_{\mu}=\left(l^{-1} q_{t}, q_{\phi}\right)$, and $\varphi_{\mu}=\left(l^{-1} \varphi_{t}, \varphi_{\phi}\right)$.

The integrability condition of the energy then reads

$$
\delta^{2} M=-\frac{1}{l}\left(\delta \varphi_{\mu} \wedge \delta q^{\mu}\right)=0
$$

which means that $\varphi_{\mu}$ and $q_{\mu}$ are functionally related. The condition (2.19) is solved by

$$
\varphi_{\mu}=-\frac{\delta \mathcal{V}}{\delta q^{\mu}}
$$

where $\mathcal{V}=\mathcal{V}\left(q^{\mu}\right)$ is an arbitrary function of $q_{t}$ and $q_{\phi}$.

Therefore, the mass and the angular momentum read

$$
\begin{aligned}
M & =f_{\mathcal{R}}+f_{\mathcal{F}}+h_{\mathcal{R}}+\frac{1}{l}\left(\mathcal{V}-q_{\phi} \frac{\delta \mathcal{V}}{\delta q_{\phi}}\right), \\
J & =j+\frac{l}{4 \pi} q_{t} q_{\phi}+q_{t} \frac{\delta \mathcal{V}}{\delta q_{\phi}}
\end{aligned}
$$

which manifestly acquire contributions from the electromagnetic field, as well as from the function $\mathcal{V}$ that characterizes the set of boundary conditions that are compatible with integrability of the energy.

It is worth highlighting that, unlike the case of higher dimensional spacetimes, for $d=3$ dimensions the precise value of the mass and the angular momentum explicitly depends on the choice of boundary conditions. Note that a similar effect is known to occur in the case of scalar fields with slow fall-off at infinity [3]. Nonetheless, in the latter case, this effect manifests only in the mass, but not in the angular momentum.

\subsection{Compatibility of the boundary conditions with Lorentz and scaling sym- metries}

As in the case of the self-adjoint extensions in quantum mechanics, it is natural to wonder about suitable boundary conditions that are consistent with a well-defined energy spectrum. In order to have a guide, it is compulsory to explore whether the set of boundary conditions is compatible with the symmetries of stationary circularly symmetric configurations of the 
form (2.2) and (2.3). Thus, since a consistent set of boundary conditions turns out to be specified by a single function $\mathcal{V}=\mathcal{V}\left(q^{\mu}\right)$, requiring invariance of them under the Lorentz boosts of the (conformally) flat boundary metric, implies that the allowed function must be of the form

$$
\mathcal{V}=\mathcal{V}\left(q^{2}\right)
$$

with $q^{2}=\eta^{\mu \nu} q_{\mu} q_{\nu}=q_{\phi}^{2}-q_{t}^{2}$. Note that the simplest choice of Lorentz-invariant boundary conditions corresponds to $\mathcal{V}=\mathcal{V}_{0}$, where $\mathcal{V}_{0}$ is an arbitrary fixed constant without variation, which can always be set to zero due to the arbitrariness in the choice of the energy of the reference background.

As explained in section 2, the class of stationary circularly symmetric configurations is invariant under scalings of the form (2.4), (2.5). Indeed, the reduced action (2.6) in the bulk scales as $I \rightarrow \lambda^{2} I$, so that the field equations are invariant under the scaling symmetry. ${ }^{2}$ Thus, it is also interesting to look for the set of "holographic boundary conditions" that is compatible with this scaling symmetry. The precise form of the function $\mathcal{V}\left(q^{\mu}\right)$ can then be found taking into account that $\varphi_{\mu}$ and $q_{\mu}$ are functionally related and transform in a different way under the scaling symmetry. The transformation rules of $\varphi_{\mu}$ and $q_{\mu}$ are inherited from the ones of the radial coordinate $r$ and the gauge field $A$, which according to (2.4) and (2.5), are given by $r \rightarrow \lambda r$, and $\mathcal{A}_{\mu} \rightarrow \lambda \mathcal{A}_{\mu}$, and hence

$$
\varphi_{\mu} \rightarrow \lambda\left(\varphi_{\mu}+\frac{l}{2 \pi} q_{\mu} \log (\lambda)\right) ; \quad q_{\mu} \rightarrow \lambda q_{\mu} .
$$

The functional relationship between $\varphi_{\mu}$ and $q_{\mu}$ then implies that

$$
\varphi_{\mu}\left(\lambda q_{\mu}\right)=\lambda\left(\varphi_{\mu}+\frac{l}{2 \pi} q_{\mu} \log (\lambda)\right) .
$$

Taking the derivative of (2.24) with respect to $\lambda$, and evaluating it for $\lambda=1$, yields the following linear differential equation

$$
q^{\nu} \frac{\partial^{2} \mathcal{V}}{\partial q^{\nu} \partial q^{\mu}}=\frac{\partial \mathcal{V}}{\partial q^{\mu}}-\frac{l}{2 \pi} q_{\mu}
$$

where $\mathcal{V}$ is defined as in eq. (2.20). The general solution is given by

$$
\mathcal{V}=q_{t}^{2} F\left(\frac{q_{\phi}}{q_{t}}\right)+\frac{l}{8 \pi}\left(q_{t}^{2}\left[\log \left(q_{t}^{2}\right)-1\right]-q_{\phi}^{2}\left[\log \left(q_{\phi}^{2}\right)-1\right]\right)
$$

up to an arbitrary constant without variation.

The set of holographic boundary conditions that is compatible with the scaling symmetry is then determined by (2.25), being described by an arbitrary function of a single variable $F=F\left(q_{\phi} / q_{t}\right)$. Note that, as expected, the function $\mathcal{V}$ in $(2.25)$ transforms anomalously under scalings, i.e., $\mathcal{V}\left(\lambda q_{\mu}\right)=\lambda^{2}\left(\mathcal{V}-\frac{q^{2} l}{4 \pi} \log (\lambda)\right)$.

Interestingly, if one requires simultaneously both Lorentz and scaling symmetries, then consistency of (2.23) with (2.25) fixes the form of the arbitrary function according to

$$
F(x)=\frac{l}{8 \pi}\left[\log \left[\frac{\kappa}{8 \pi^{2}}\left(1-x^{2}\right)\right]-x^{2} \log \left[\frac{\kappa}{8 \pi^{2}}\left(x^{-1}-1\right)\right]+\gamma\left(1-x^{2}\right)\right],
$$

\footnotetext{
${ }^{2} \mathrm{~A}$ similar scaling symmetry, for which the reduced action is invariant has been reported in [26].
} 
where $\gamma$ is an arbitrary fixed constant. Therefore, the holographic Lorentz invariant set of boundary conditions corresponds to the following choice:

$$
\mathcal{V}=\frac{l}{8 \pi}\left(q_{t}^{2}-q_{\phi}^{2}\right)\left[\log \left(\frac{\kappa}{8 \pi^{2}}\left(q_{t}^{2}-q_{\phi}^{2}\right)\right)+\gamma-1\right]
$$

\section{Black hole spectrum and Lorentz invariant boundary conditions: sim- plest and holographic choices}

Let us focus on the analysis of global charges in the case of electrically charged rotating black hole solutions for a generic choice of boundary conditions. We then concentrate in Lorentz invariant choices for the simplest, as well as for the holographic cases. For the sake of simplicity, we begin describing the static solution, and then we extend to the rotating case.

\subsection{Static electrically charged black hole}

The static electrically charged solution found in [9] can be written as

$$
\begin{aligned}
d s^{2} & =-N_{\infty}^{2}\left(\frac{r^{2}}{l^{2}}-\frac{r_{+}^{2}}{l^{2}}-\frac{\kappa q_{t}^{2}}{4 \pi^{2}} \log \left(\frac{r}{r_{+}}\right)\right) d t^{2}+\frac{d r^{2}}{\frac{r^{2}}{l^{2}}-\frac{r_{+}^{2}}{l^{2}}-\frac{\kappa q_{t}^{2}}{4 \pi^{2}} \log \left(\frac{r}{r_{+}}\right)}+r^{2} d \phi^{2} \\
A & =\left(-\frac{q_{t}}{2 \pi} N_{\infty} \log \left(\frac{r}{l}\right)+N_{\infty} \frac{\varphi_{t}}{l}-\Phi\right) d t
\end{aligned}
$$

where the event horizon locates at $r=r_{+}$, provided the electric charge is bounded as

$$
q_{t}^{2} \leq \frac{8 \pi^{2}}{\kappa l^{2}} r_{+}^{2}
$$

This bound saturates in the extremal case. According to (2.14), the asymptotic behaviour is such that $q_{\phi}=\varphi_{\phi}=h_{\mathcal{R}}=f_{\mathcal{R}}=0$, and

$$
f_{\mathcal{F}}=\frac{\pi r_{+}^{2}}{\kappa l^{2}}-\frac{q_{t}^{2}}{4 \pi} \log \left(\frac{r_{+}}{l}\right)
$$

so that the global charges can be readily found from (2.21), (2.22).

For a generic choice of $\mathcal{V}=\mathcal{V}\left(q_{t}\right)$ the angular momentum vanishes, and the mass reduces to

$$
M=\frac{\pi r_{+}^{2}}{\kappa l^{2}}-\frac{q_{t}^{2}}{4 \pi} \log \left(\frac{r_{+}}{l}\right)+\frac{1}{l} \mathcal{V}
$$

Simplest Lorentz invariant boundary conditions. In the case of $\mathcal{V}=0$, the result in (3.3) agrees with the one found in [2]. Note that in the extremal case, the mass reads

$$
M_{\mathrm{ext}}=\frac{q_{t}^{2}}{8 \pi}\left[1-\log \left(\frac{\kappa q_{t}^{2}}{8 \pi^{2}}\right)\right] .
$$

As explained in [2], the energy spectrum is unbounded from below, and for a fixed value of the mass, the electric charge possesses no upper bound, see figure 1(a). 




Figure 1. Electrically charged black holes exist in the region of the $\left(M, q_{t}^{2}\right)$-plane defined through $M \geq M_{\text {ext }}$, being delimited by the curve that corresponds to the extremal solution. Figure (a) describes the standard case, that corresponds to the simplest choice of Lorentz invariant boundary conditions: $\mathcal{V}=0$, so that $M_{\text {ext }}=\frac{q_{t}^{2}}{8 \pi}\left[1-\log \left(\frac{\kappa q_{t}^{2}}{8 \pi^{2}}\right)\right]$. The energy spectrum is unbounded from below, and for a fixed value of the mass there is no upper bound on the electric charge. Figure (b) illustrates the case of holographic Lorentz invariant boundary conditions: $\mathcal{V}=\frac{l}{8 \pi} q_{t}^{2}\left[\log \left(\frac{\kappa}{8 \pi^{2}} q_{t}^{2}\right)+\gamma-1\right]$, so that $M_{\text {ext }}=\frac{\gamma}{8 \pi} q_{t}^{2}$, for $\gamma>0$. The energy spectrum is nonnegative, and for a fixed value of the mass the electric charge is bounded from above.

Holographic Lorentz invariant boundary conditions. In this case, according to (2.26), the suitable boundary conditions that are consistent with the scaling symmetry are determined by

$$
\mathcal{V}=\frac{l}{8 \pi} q_{t}^{2}\left[\log \left(\frac{\kappa}{8 \pi^{2}} q_{t}^{2}\right)+\gamma-1\right]
$$

which depends on a single fixed parameter $\gamma$. The black hole mass in (3.3) then reads

$$
M=\frac{\pi r_{+}^{2}}{\kappa l^{2}}+\frac{q_{t}^{2}}{8 \pi}\left[\log \left(\frac{\kappa l^{2}}{8 \pi^{2}} \frac{q_{t}^{2}}{r_{+}^{2}}\right)+\gamma-1\right]
$$

so that in the extremal case is given by

$$
M_{\mathrm{ext}}=\frac{\gamma}{8 \pi} q_{t}^{2}
$$

Remarkably if the arbitrary parameter fulfills $\gamma>0$, the spectrum is such that the energy is nonnegative, and for a fixed value of the mass, the electric charge is bounded from above. This is depicted in figure 1(b). For $\gamma=0$, the energy spectrum remains nonnegative, but the electric charge has no upper bound. The case $\gamma<0$ is pathological, since the energy is unbounded from below and there is no upper bound for the electric charge.

\subsection{Electrically charged rotating black hole}

The electrically charged rotating black hole solution has been obtained in [2], and independently in [27] following a different approach. The spacetime metric and the gauge field 
can be written as in eqs. (2.2), (2.3), with

$$
\begin{aligned}
\mathcal{R}^{2} & =r^{2}+\left(\frac{\omega^{2}}{1-\omega^{2}}\right) r_{+}^{2}+\frac{\kappa}{4 \pi^{2}}\left(q_{t} \omega l\right)^{2} \log \left(\frac{r}{r_{+}}\right), \\
\mathcal{N}^{\phi} & =N_{\infty}^{\phi}-\left(\frac{\omega}{1-\omega^{2}}\right)\left(\frac{r^{2}}{l^{2}}-\mathcal{F}^{2}\right) \frac{l}{\mathcal{R}^{2}} N_{\infty}, \\
\mathcal{N}^{2} & =\frac{r^{2}}{\mathcal{R}^{2}} N_{\infty}^{2} \\
\mathcal{F}^{2} & =\frac{r^{2}}{l^{2}}-\frac{r_{+}^{2}}{l^{2}}-\frac{\kappa}{4 \pi^{2}} q_{t}^{2}\left(1-\omega^{2}\right) \log \left(\frac{r}{r_{+}}\right), \\
\mathcal{A}_{t} & =-\frac{q_{t}}{2 \pi}\left[N_{\infty}-\omega l N_{\infty}^{\phi}\right] \log \left(\frac{r}{l}\right)+N_{\infty}^{\phi} \varphi_{\phi}+N_{\infty} \frac{\varphi_{t}}{l}-\Phi, \\
\mathcal{A}_{\phi} & =\frac{q_{t} \omega l}{2 \pi} \log \left(\frac{r}{l}\right)+\varphi_{\phi} .
\end{aligned}
$$

This configuration possesses an event horizon at $r=r_{+}$, provided the electric charge $q_{t}$ and the rotation parameter $\omega$ fulfill the following bounds

$$
\begin{aligned}
\omega^{2} & \leq 1, \\
q_{t}^{2} & \leq \frac{8 \pi^{2}}{\kappa l^{2}} \frac{r_{+}^{2}}{1-\omega^{2}},
\end{aligned}
$$

that saturate in the extremal cases. The relevant contributions to the global charges in (2.21), (2.22) can be directly read from the asymptotic behaviour in (2.14), which are determined by

$$
\begin{aligned}
h_{\mathcal{R}} & =-\frac{\omega^{2} q_{t}^{2}}{4 \pi} ; & f_{\mathcal{R}} & =\frac{2 \pi}{\kappa l^{2}} \frac{r_{+}^{2} \omega^{2}}{1-\omega^{2}}-\frac{q_{t}^{2} \omega^{2}}{2 \pi} \log \left(\frac{r_{+}}{l}\right), \\
q_{\phi} & =-q_{t} \omega ; & f_{\mathcal{F}} & =\frac{\pi r_{+}^{2}}{\kappa l^{2}}-\frac{q_{t}^{2}\left(1-\omega^{2}\right)}{4 \pi} \log \left(\frac{r_{+}}{l}\right) .
\end{aligned}
$$

Hence, for a generic choice of boundary conditions given by $\mathcal{V}=\mathcal{V}\left(q^{\mu}\right)$, the mass and the angular momentum read

$$
\begin{aligned}
M & =\frac{\pi r_{+}^{2}}{\kappa l^{2}}\left(\frac{1+\omega^{2}}{1-\omega^{2}}\right)-\frac{q_{t}^{2}}{4 \pi}\left(\omega^{2}+\left(1+\omega^{2}\right) \log \left(\frac{r_{+}}{l}\right)\right)+\frac{1}{l}\left(\mathcal{V}-q_{\phi} \frac{\delta \mathcal{V}}{\delta q_{\phi}}\right), \\
J & =\frac{2 \pi r_{+}^{2} \omega}{\kappa l\left(1-\omega^{2}\right)}-\frac{q_{t}^{2} \omega l}{4 \pi}\left(1+\log \left(\frac{r_{+}^{2}}{l^{2}}\right)\right)+q_{t} \frac{\delta \mathcal{V}}{\delta q_{\phi}}
\end{aligned}
$$

respectively.

Note that for a generic choice of boundary conditions, the value of the mass and the angular momentum might be sensitive to the sign of the electric charge, or even the sign of the angular momentum could be the opposite of the rotation parameter, which are certainly curious but not necessarily inconsistent features. ${ }^{3}$ In this sense, despite the

\footnotetext{
${ }^{3}$ Indeed, the sign of $J$ can differ from the one of $\omega$ for black holes in gravity theories with parity odd terms in the action, like in the case of topologically massive gravity (see, e.g. [28]). Besides, as it has been recently shown in [29], in the case of black holes on $\mathrm{AdS}_{3}$ endowed with spin-four fields, the allowed range of positive spin-four charges is wider than that of the negative ones. Furthermore, the allowed range of spin-four charges is consistent, and precisely agrees, with the one that comes from the bounds that are obtained from the locally hypersymmetric extension of the theory.
} 
global charges have acquired explicit contributions due to presence of the arbitrary function $\mathcal{V}$, in the (naive) limit of extreme rotation $\left(\omega^{2} \rightarrow 1\right)$ they relate as in the electrically neutral case $[9,30]$, i.e., $J / M l=1$. It is also amusing to perform an explicit check of the validity of the first law of thermodynamics when the global charges are generically given by (3.10), (3.11), which is known to hold in advance because it is just reflection of the fact that the Euclidean reduced action attains an extremum for smooth solutions. Indeed, demanding regularity of the Euclidean geometry and the gauge field around the event horizon implies that

$$
\begin{aligned}
N_{\infty}^{2} & =\frac{4 \pi^{2} l^{4} r_{+}^{2}}{1-\omega^{2}}\left(r_{+}^{2}-\frac{\kappa l^{2}}{8 \pi^{2}} q_{t}^{2}\left(1-\omega^{2}\right)\right)^{-2} \\
N_{\infty}^{\phi} & =\frac{\omega}{l} N_{\infty} \\
\Phi & =-\frac{q_{t}}{2 \pi}\left(N_{\infty}-\omega l N_{\infty}^{\phi}\right) \log \left(\frac{r_{+}}{l}\right)-N_{\infty}^{\phi} \frac{\delta \mathcal{V}}{\delta q_{\phi}}+\frac{1}{l} N_{\infty} \frac{\delta \mathcal{V}}{\delta q_{t}},
\end{aligned}
$$

so that the variation of the entropy, $S=\frac{A}{4 G}=\frac{\pi \mathcal{R}\left(r_{+}\right)}{2 G}$, fulfills

$$
\delta S=N_{\infty} \delta M-N_{\infty}^{\phi} \delta J-\Phi \delta q_{t}
$$

Therefore, $N_{\infty}$ corresponds to the inverse Hawking temperature $\beta=T^{-1}$, while the product of $\beta$ times the chemical potentials associated to the angular momentum and the electric charge are identified with $N_{\infty}^{\phi}$, and $\Phi$, respectively.

Lorentz invariant boundary conditions. Requiring the boundary conditions to be consistent with Lorentz invariance at the boundary implies that $\mathcal{V}=\mathcal{V}\left(q^{2}\right)$. It is worth pointing out that, according to eqs. (2.21) and (2.22), this ensures that the angular momentum possesses the same sign as the rotation parameter, and also guarantees that both the mass and the angular momentum do not depend on the sign of the electric charge $q_{t}$. Note that in the simplest case, $\mathcal{V}=0$, expressions (3.10) and (3.11) agree with the results found in [2].

Holographic Lorentz invariant boundary conditions. The boundary conditions in (2.26) in this case read

$$
\mathcal{V}=\frac{l}{8 \pi} q_{t}^{2}\left(1-\omega^{2}\right)\left[\log \left(\frac{\kappa}{8 \pi^{2}} q_{t}^{2}\left(1-\omega^{2}\right)\right)+\gamma-1\right],
$$

so that the mass and the angular momentum in (3.10) and (3.11) reduce to

$$
\begin{aligned}
M & =\frac{\pi}{\kappa}\left(\frac{1+\omega^{2}}{1-\omega^{2}}\right) \frac{r_{+}^{2}}{l^{2}}+\frac{q_{t}^{2}\left(1+\omega^{2}\right)}{8 \pi}\left(\log \left[\frac{\kappa}{8 \pi^{2}} \frac{q_{t}^{2} l^{2}}{r_{+}^{2}}\left(1-\omega^{2}\right)\right]+\gamma-1\right), \\
J & =\frac{2 l \omega}{1+\omega^{2}} M .
\end{aligned}
$$

Interestingly, the relationship in (3.18) does not involve the electric charge, and hence, it precisely agrees with the one for the electrically neutral BTZ black hole. In the extremal 
case for which the bound (3.9) saturates, the black hole mass reads

$$
M_{\mathrm{ext}}=\frac{\gamma}{8 \pi} q_{t}^{2}\left(1+\omega^{2}\right) .
$$

It is then clear that the energy spectrum and the upper bound in the electric charge remain well-behaved also in the rotating case.

\section{Final remarks}

We have shown that the mass and the angular momentum of stationary circularly symmetric solutions of the Einstein-Maxwell theory on $\mathrm{AdS}_{3}$ generically acquire nontrivial contributions due to the electromagnetic field, and turn out to be sensitive to the choice of boundary conditions. Indeed, this effect not only manifests for spin-1 fields, since it is known that it also occurs for scalar [3, 31, 32] and even for higher spin fields [33] in three spacetime dimensions. It is worth pointing out that, according to different results found in the literature $[2,13,19,34-40]$, the precise value of the electrically charged black hole mass manifestly appears to depend on the distinct regularization procedures. In this sense, our results might shed light on this puzzle, since the different results could just correspond to inequivalent choices of boundary conditions. It would also be interesting to explore the effect of different choices of the function $\mathcal{V}$ that defines the suitable boundary conditions in the context of holographic superconductivity [41-46].

There is a very special choice of boundary conditions that is singled out by requiring compatibility with Lorentz and scaling symmetries. This set of "holographic boundary conditions" is characterized by a unique fixed parameter $\gamma$, that plays a similar role as the length of the box for a confined free particle in quantum mechanics, or a modulus parameter in a gauge field theory. The holographic boundary conditions can also be naturally interpreted as an analog of Robin boundary conditions. It is worth highlighting that this parameter manifestly appears in the energy spectrum of rotating black hole solutions, which for $\gamma>0$ is nonnegative, and for a fixed energy level there is an upper bound for the electric charge. One then naturally expects that the good properties of the energy spectrum should be inherited to different aspects of the thermodynamic structure. Jumping ahead, it would also be worth to reconsider whether electrically charged black holes could be suitably embedded within an appropriate supergravity theory. In this case, one should expect that the extremal case would saturate the energy bounds that come from supersymmetry. Note that, according to eq. (3.6), the energy bound should be quadratic in the electric charge, which appears to go by hand with the quadratic nonlinearity of the superconformal algebra with $\mathcal{N}>1$ (see e.g. [47] and references therein).

As an ending remark we would like to mention that the Brown-Henneaux boundary conditions [5] can be consistently relaxed so as to accommodate electrically charged black holes in the Einstein-Maxwell theory on $\mathrm{AdS}_{3}$ [48]. Remarkably, the asymptotic behaviour can be further relaxed, so as to accommodate the generic rotating case; and if one requires these fall-off conditions to be mapped into themselves under the full conformal group in two dimensions, the holographic choice of boundary conditions in (2.26) turns out to be singled out [49]. 


\section{Acknowledgments}

We thank Glenn Barnich, Claudio Bunster, Oscar Fuentealba, Marc Henneaux, Cristián Martínez, and Jorge Zanelli for helpful comments and discussions. We are indebted to Hernán González for sharing his insights in an early stage of this work. M.R. thanks Conicyt for financial support. The work of D.T. is partially supported by the ERC through the "SyDuGraM" Advanced Grant, by FNRS-Belgium (convention FRFC PDR T.1025.14 and convention IISN 4.4503.15) and by the "Communauté Française de Belgique" through the ARC program. This research has been partially supported by Fondecyt grants № 11130262, 11130260, 1130658, 1121031. Centro de Estudios Científicos (CECs) is funded by the Chilean Government through the Centers of Excellence Base Financing Program of Conicyt.

Open Access. This article is distributed under the terms of the Creative Commons Attribution License (CC-BY 4.0), which permits any use, distribution and reproduction in any medium, provided the original author(s) and source are credited.

\section{References}

[1] S. Deser and P.O. Mazur, Static Solutions in D = 3 Einstein-Maxwell Theory, Class. Quant. Grav. 2 (1985) L51 [INSPIRE].

[2] C. Martinez, C. Teitelboim and J. Zanelli, Charged rotating black hole in three space-time dimensions, Phys. Rev. D 61 (2000) 104013 [hep-th/9912259] [INSPIRE].

[3] M. Henneaux, C. Martinez, R. Troncoso and J. Zanelli, Black holes and asymptotics of $2+1$ gravity coupled to a scalar field, Phys. Rev. D 65 (2002) 104007 [hep-th/0201170] [InSPIRE].

[4] C. Bunster and A. Pérez, Superselection rule for the cosmological constant in three-dimensional spacetime, Phys. Rev. D 91 (2015) 024029 [arXiv:1412.1492] [InSPIRE].

[5] J.D. Brown and M. Henneaux, Central Charges in the Canonical Realization of Asymptotic Symmetries: An Example from Three-Dimensional Gravity, Commun. Math. Phys. 104 (1986) 207 [INSPIRE].

[6] O. Coussaert and M. Henneaux, Supersymmetry of the (2+1) black holes, Phys. Rev. Lett. 72 (1994) 183 [hep-th/9310194] [INSPIRE].

[7] A. Chatrabhuti, F. Englert, L. Houart and A. Taormina, Fermionic subspaces of the bosonic string, Class. Quant. Grav. 20 (2003) S449 [hep-th/0212085] [INSPIRE].

[8] J.R. Gott, J.Z. Simon and M. Alpert, General Relativity in a (2+1)-dimensional Space-time: An Electrically Charged Solution, Gen. Rel. Grav. 18 (1986) 1019 [InSPIRE].

[9] M. Bañados, C. Teitelboim and J. Zanelli, The black hole in three-dimensional space-time, Phys. Rev. Lett. 69 (1992) 1849 [hep-th/9204099] [INSPIRE].

[10] P. Peldan, Unification of gravity and Yang-Mills theory in (2+1)-dimensions, Nucl. Phys. B 395 (1993) 239 [gr-qc/9211014] [INSPIRE].

[11] M. Kamata and T. Koikawa, The electrically charged BTZ black hole with self (antiself) dual Maxwell field, Phys. Lett. B 353 (1995) 196 [hep-th/9505037] [INSPIRE]. 
[12] K.C.K. Chan, Comment on the calculation of the angular momentum for the (anti)selfdual charged spinning BTZ black hole, Phys. Lett. B 373 (1996) 296 [gr-qc/9509032] [INSPIRE].

[13] G. Clement, Spinning charged BTZ black holes and selfdual particle-like solutions, Phys. Lett. B 367 (1996) 70 [gr-qc/9510025] [INSPIRE].

[14] E.W. Hirschmann and D.L. Welch, Magnetic solutions to (2+1) gravity, Phys. Rev. D 53 (1996) 5579 [hep-th/9510181] [INSPIRE].

[15] M. Cataldo and P. Salgado, Static Einstein-Maxwell solutions in (2+1)-dimensions, Phys. Rev. D 54 (1996) 2971 [INSPIRE].

[16] M. Kamata and T. Koikawa, (2+1)-dimensional charged black hole with (anti-)selfdual Maxwell fields, Phys. Lett. B 391 (1997) 87 [hep-th/9605114] [INSPIRE].

[17] M. Cataldo and P. Salgado, Three dimensional extreme black hole with self (anti-self) dual Maxwell field, Phys. Lett. B 448 (1999) 20 [InSPIRE].

[18] M. Cataldo, Azimuthal electric field in a static rotationally symmetric (2+1)-dimensional space-time, Phys. Lett. B 529 (2002) 143 [gr-qc/0201047] [InSPIRE].

[19] O.J.C. Dias and J.P.S. Lemos, Rotating magnetic solution in three-dimensional Einstein gravity, JHEP 01 (2002) 006 [hep-th/0201058] [INSPIRE].

[20] M. Cataldo, J. Crisostomo, S. del Campo and P. Salgado, On magnetic solution to $(2+1)$ Einstein-Maxwell gravity, Phys. Lett. B 584 (2004) 123 [hep-th/0401189] [INSPIRE].

[21] J. Matyjasek and O.B. Zaslavskii, Extremal limit for charged and rotating (2+1)-dimensional black holes and Bertotti-Robinson geometry, Class. Quant. Grav. 21 (2004) 4283 [gr-qc/0404090] [INSPIRE].

[22] A.A. Garcia-Diaz, Three dimensional stationary cyclic symmetric Einstein-Maxwell solutions; black holes, Annals Phys. 324 (2009) 2004 [arXiv:1307.6655] [INSPIRE].

[23] E. Ayon-Beato, M. Cataldo and A.A. Garcia, Electromagnetic fields in stationary cyclic symmetric $2+1$ gravity, at 10th International Symposium on Particles, Strings and Cosmology (PASCOS 04 and Pran Nath Fest), Boston U.S.A. (2004).

[24] M. Henneaux and C. Teitelboim, The cosmological constant as a canonical variable, Phys. Lett. B 143 (1984) 415 [INSPIRE].

[25] T. Regge and C. Teitelboim, Role of Surface Integrals in the Hamiltonian Formulation of General Relativity, Annals Phys. 88 (1974) 286 [INSPIRE].

[26] M. Bañados and S. Theisen, Scale invariant hairy black holes, Phys. Rev. D 72 (2005) 064019 [hep-th/0506025] [INSPIRE].

[27] G. Clement, Classical solutions in three-dimensional Einstein-Maxwell cosmological gravity, Class. Quant. Grav. 10 (1993) L49 [INSPIRE].

[28] M. Henneaux, C. Martinez and R. Troncoso, Asymptotically anti-de Sitter spacetimes in topologically massive gravity, Phys. Rev. D 79 (2009) 081502 [arXiv:0901.2874] [InSPIRE].

[29] M. Henneaux, A. Perez, D. Tempo and R. Troncoso, Hypersymmetry bounds and three-dimensional higher-spin black holes, JHEP 08 (2015) 021 [arXiv:1506.01847] [INSPIRE].

[30] M. Bañados, M. Henneaux, C. Teitelboim and J. Zanelli, Geometry of the (2+1) black hole, Phys. Rev. D 48 (1993) 1506 [Erratum ibid. D 88 (2013) 069902] [gr-qc/9302012] [INSPIRE]. 
[31] M. Henneaux, C. Martinez, R. Troncoso and J. Zanelli, Asymptotically anti-de Sitter spacetimes and scalar fields with a logarithmic branch, Phys. Rev. D 70 (2004) 044034 [hep-th/0404236] [INSPIRE].

[32] M. Cardenas, O. Fuentealba and C. Martínez, Three-dimensional black holes with conformally coupled scalar and gauge fields, Phys. Rev. D 90 (2014) 124072 [arXiv: 1408.1401] [INSPIRE].

[33] A. Perez, D. Tempo and R. Troncoso, Higher spin gravity in 3D: Black holes, global charges and thermodynamics, Phys. Lett. B 726 (2013) 444 [arXiv:1207.2844] [INSPIRE].

[34] G. Clement, Black hole mass and angular momentum in 2+1 gravity, Phys. Rev. D 68 (2003) 024032 [gr-qc/0301129] [inSPIRE].

[35] M. Cadoni, M. Melis and M.R. Setare, Microscopic entropy of the charged BTZ black hole, Class. Quant. Grav. 25 (2008) 195022 [arXiv:0710.3009] [INSPIRE].

[36] Y.S. Myung, Y.-W. Kim and Y.-J. Park, Entropy function approach to charged BTZ black hole, Gen. Rel. Grav. 42 (2010) 1919 [arXiv:0903.2109] [InSPIRE].

[37] K. Jensen, Chiral anomalies and AdS/CMT in two dimensions, JHEP 01 (2011) 109 [arXiv: 1012.4831] [INSPIRE].

[38] A.A. Garcia-Diaz, Three dimensional stationary cyclic symmetric Einstein-Maxwell solutions; energy, mass, momentum and algebraic tensors characteristics, arXiv:1307.6652 [INSPIRE].

[39] G. Barnich, P.-H. Lambert and P. Mao, Three-dimensional asymptotically flat Einstein-Maxwell theory, arXiv:1503.00856 [INSPIRE].

[40] S.H. Hendi, S. Panahiyan and R. Mamasani, Thermodynamic stability of charged BTZ black holes: Ensemble dependency problem and its solution, Gen. Rel. Grav. 47 (2015) 91 [arXiv: 1507.08496] [INSPIRE].

[41] S.A. Hartnoll, C.P. Herzog and G.T. Horowitz, Building a Holographic Superconductor, Phys. Rev. Lett. 101 (2008) 031601 [arXiv:0803.3295] [INSPIRE].

[42] D. Maity, S. Sarkar, N. Sircar, B. Sathiapalan and R. Shankar, Properties of CFTs dual to Charged BTZ black-hole, Nucl. Phys. B 839 (2010) 526 [arXiv:0909.4051] [InSPIRE].

[43] J. Ren, One-dimensional holographic superconductor from $A d S_{3} / C F T_{2}$ correspondence, JHEP 11 (2010) 055 [arXiv: 1008.3904] [INSPIRE].

[44] T. Faulkner and N. Iqbal, Friedel oscillations and horizon charge in 1D holographic liquids, JHEP 07 (2013) 060 [arXiv:1207.4208] [INSPIRE].

[45] G.T. Horowitz, N. Iqbal and J.E. Santos, Simple holographic model of nonlinear conductivity, Phys. Rev. D 88 (2013) 126002 [arXiv:1309.5088] [INSPIRE].

[46] P. Chaturvedi and G. Sengupta, Rotating BTZ Black Holes and One Dimensional Holographic Superconductors, Phys. Rev. D 90 (2014) 046002 [arXiv:1310.5128] [InSPIRE].

[47] M. Henneaux, L. Maoz and A. Schwimmer, Asymptotic dynamics and asymptotic symmetries of three-dimensional extended AdS supergravity, Annals Phys. 282 (2000) 31 [hep-th/9910013] [inSPIRE].

[48] G. Barnich and P.-H. Lambert, Einstein-Yang-Mills theory: Asymptotic symmetries, Phys. Rev. D 88 (2013) 103006 [arXiv:1310.2698] [INSPIRE].

[49] A. Pérez, M. Riquelme, D. Tempo and R. Troncoso, Asymptotic structure of the Einstein-Maxwell theory on $A d S_{3}$, Preprint CECS-PHY-15/08. 